\title{
Computational identification of miRNAs that modulate the differentiation of mesenchymal stem cells to osteoblasts
}

Kanokwan Seenprachawong, Pornlada Nuchnoi, Chanin Nantasenamat, Virapong Prachayasittikul, Aungkura Supokawej

MicroRNAs (miRNAs) are small endogenous noncoding RNAs that play an instrumental role in post-transcriptional modulation of gene expression. Genes related to osteogenesis (i.e. $R U N X 2, C O L 1 A 1$ and $O S X)$ is important in controlling the differentiation of mesenchymal stem cells (MSCs) to bone tissues. The regulated expression level of miRNAs is critically important for the differentiation of MSCs to preosteoblasts. The understanding of miRNA regulation in osteogenesis could be applied for future applications in bone defects.

Therefore, this study aims to shed light on the mechanistic pathway underlying osteogenesis by predicting miRNAs that may modulate this pathway. This study investigates RUNX2, which is a major transcription factor for osteogenesis that drives MSCs into preosteoblasts. Three different prediction tools were employed for identifying miRNAs related to osteogenesis using the 3'UTR of RUNX2 as the target gene. Of the 1,023 miRNAs, 70 miRNAs were found by at least two of the tools. Candidate miRNAs were then selected based on their free energy values, followed by assessing the probability of target accessibility. The results showed that miRNAs 23b, 23a, 30b, 143, 203, 217, and 221 could regulate the RUNX2 gene during the differentiation of MSCs to preosteoblasts. 


\title{
Computational identification of miRNAs
}

that modulate the differentiation of

mesenchymal stem cells to osteoblasts

\author{
4anokwan Seenprachawong ${ }^{1}$, Pornlada Nuchnoi ${ }^{2}$, \\ Chanin Nantasenamat ${ }^{3}$, Virapong Prachayasittikul ${ }^{4}$, and \\ Aungkura Supokawej*1 \\ ${ }^{1}$ Department of Clinical Microscopy, Faculty of Medical Technology, Mahidol University, \\ Bangkok 10700, Thailand \\ ${ }^{2}$ Center for Research and Innovation, Faculty of Medical Technology, Mahidol \\ University, Bangkok 10700, Thailand \\ ${ }^{3}$ Center of Data Mining and Biomedical Informatics, Faculty of Medical Technology, \\ Mahidol University, Bangkok 10700, Thailand \\ ${ }^{4}$ Department of Clinical Microbiology and Applied Technology, Faculty of Medical \\ Technology, Mahidol University, Bangkok 10700, Thailand
}

15

\section{ABSTRACT}

MicroRNAs (miRNAs) are small endogenous noncoding RNAs that play an instrumental role in posttranscriptional modulation of gene expression. Genes related to osteogenesis (e.g. RUNX2, COL1A1 and $O S X$ ) is important in controlling the differentiation of mesenchymal stem cells (MSCs) to bone tissues. The regulated expression level of miRNAs is critically important for the differentiation of MSCs to preosteoblasts. The understanding of miRNA regulation in osteogenesis could be applied for future applications in bone defects. Therefore, this study aims to shed light on the mechanistic pathway underlying osteogenesis by predicting miRNAs that may modulate this pathway. This study investigates RUNX2, which is a major transcription factor for osteogenesis that drives MSCs into preosteoblasts. Three different prediction tools were employed for identifying miRNAs related to osteogenesis using the 3'UTR of RUNX2 as the target gene. Of the 1,023 miRNAs, 70 miRNAs were found by at least two of the tools. Candidate miRNAs were then selected based on their free energy values, followed by assessing the probability of target accessibility. The results showed that miRNAs 23b, 23a, 30b, 143, 203, 217, and 221 could regulate the RUNX2 gene during the differentiation of MSCs to preosteoblasts.

Keywords: microRNAs, miRNAs, osteogenesis, mesenchymal stem cells, RUNX2, bioinformatics

\section{INTRODUCTION}

Osteogenesis is a complex multistep process that includes proliferation, maturation and matrix mineralization from the development of mesenchymal stem cells into bone tissue. Mesenchymal stem cells (MSCs) are a type of adult stem cell that can be isolated from bone marrow and various tissues, such as muscle, adipose tissue, placenta and umbilical cord (Erices et al., 2000). MSCs are multipotent stem cells that have the ability to self-renew or differentiate into mesodermal-derived cells, such as osteoblasts, chondrocytes and adipocytes (Deans and Moseley, 2000; Pittenger et al., 1999). MSCs have high potential for use in cell therapy due to their special properties: proinflammatory, immunoprivilege, and multi-differentiation. Most importantly, the use of pluripotent stem cells derived from embryonic stem cells (ESCs) or induced pluripotent stem cells (iPSCs) is still limited because of ethical issues, technical problems and teratoma formation of these cells. Transplantation of these MSCs has clear advantages for the future treatment of bone defects, bone fractures, osteoporosis and osteoarthritis according to both in vitro studies and clinical trials. The potential of treatment showed a low outcome due to many unknown mechanisms of MSCs, particularly the osteogenic regulatory system of MSCs. There are many signaling pathways, such

*Corresponding author. E-mail: aungkura.jer@mahidol.ac.th 
as the Wnt signaling pathway and BMP pathway, that play an integrative role for bone development (James, 2013). These signaling pathways ultimately affect major transcription factors, such as runt-related transcription factor2 (RUNX2) and osterix (OSX) (Komori, 2006). RUNX2 is a major transcription factor that regulates the differentiation of MSCs to preosteoblasts, and osterix plays a significant role in the development of the preosteoblast stage into osteoblasts. In this work, the regulatory system of osteogenesis is extensively discussed, including not only the signaling pathway but also epigenetic control, such as DNA methylation, histone modification and miRNAs.

MicroRNAs (miRNAs) are small endogenous non-coding RNAs, and their length is approximately $21-$ 24 nucleotides. MiRNAs regulate gene expression at the post-transcription level through the degradation of mRNA or inhibition of protein synthesis (He and Hannon, 2004). Their function is through specific binding of miRNA and the 3' UTR of the target gene. MiRNAs are associated with stem cell differentiation and tissue development, including bone development. The regulation of miRNAs in osteogenesis has been studied, particularly in the expression of RUNX2. In the studies of Huang et al. (2010) and Tome et al. (2011), miR-204 and miR-335 exhibited an inhibitory mechanism through binding at the 3' untranslated region of RUNX2. In addition, miR-103a inhibited bone formation by binding the RUNX2 target under both physiological and pathological mechanical conditions during in vitro and in vivo studies (Zuo et al., 2015). Zhang et al. (2011) found that the osterix gene was regulated by miR-637. MiR-637 enhanced adipogenesis and inhibited osteogenesis.

RUNX2 is a master transcription factor that controls osteogenesis. RUNX2 or CBFA- 1 knockout mice showed a complete defect of bone formation because of osteoblast maturational arrest (Komori et al., 1997). The activation of RUNX2 in osteogenesis is regulated by several signaling pathways (i.e. Wnt and bone morphogenic protein) (Hayrapetyan et al., 2015). The epigenetic regulation of osteogenesis has been widely discussed but it is not well characterized, particularly, the mechanism of miRNAs. Recently, Kang and Hata (2015) proposed that the major mechanism of the regulatory function of miRNAs can be attributed to its controlling of the osteogenesis process via the cell fate determination of stem cells. Moreover, the functions of miRNAs are complex and remain unclear; thus, more studies on the role of miRNAs in osteogenesis are needed for future applications in clinical trials and diagnoses because previous studies cannot clearly describe the multiple steps of osteogenesis. Microarrays and direct cloning are typically used for predicting miRNAs, but these approaches are time consuming and expensive. Therefore, the objective of this study is to apply bioinformatics tools for predicting the miRNAs involved in osteogenesis, which is performed using the 3' untranslated region (3'UTR) of RUNX2 gene and miRNA database.

\section{MATERIALS AND METHODS}

\section{Data collection}

The workflow implemented for miRNA prediction is shown in Figure 1. The human 3'UTR sequence of $R U N X 2$ was obtained from the NCBI database (www.ncbi.nlm.nih.gov). Using the nucleotide database and keywords including homo sapiens, $R U N X 2$, and mRNA for searching nucleotide sequences, the results showed 122 nucleotide sequences. Homo sapiens runt-related transcription factor 2 (RUNX2), transcript variant 1, mRNA (accession number NM_001024630) was selected. It is a 5,553 bp linear mRNA. The structure of human protein coding mRNA includes a 5'cap, 5'UTR, coding sequence, 3'UTR, and a poly-A tail. The 3'UTR sequence of the RUNX2 gene is located downstream from the coding sequence region and is composed of 3,777 nucleotide bases (Supplemental Table S1).

\section{Prediction of miRNAs}

The prediction of miRNAs was investigated using 3 different algorithms that are the most widely used in the updated version as follows: miRanda, RegRNA and TargetScan.

miRanda software (Betel et al., 2010) has a miRNA prediction function that uses an algorithm called mirSVR. The mirSVR algorithm learns to predict mRNA target sites on mRNA expression changes from a panel of mRNA transfection experiments and displays the scores and ranks the efficiency of the miRanda-predicted miRNAs. mirSVR used 3 main features: 1) duplex structure of the target site and miRNA at the seed region, 2) composition flanking the target site, and 3)secondary structure accessibility of the site and conservation to calculate and display the mirSVR score. To predict miRNAs, the name of the target mRNA and species were used as the input. The lower mirSVR scores are correlated with 
down-regulation at the mRNA or protein levels and can be interpreted as a probability of target inhibition, leading to candidate miRNA selection.

RegRNA version 2.0 (Huang et al., 2006; Chang et al., 2013) is an integrated web server for miRNA prediction. This software database was retrieved from a literature survey of experimentally validated miRNAs, namely, miRBase. The miRBase database provides extensive miRNA sequence data, annotations and predicted gene targets. To predict miRNAs, the mRNA target sequence was used as the input. The prediction results are presented via both textual and graphical interfaces. The minimum free energy (MFE) of the miRNA-target site duplex and score are determined by miRanda and RegRNA integrated tools. The known miRNA genes in 3 mammalian genomes, including human, mouse and rat, were obtained from miRBase. Therefore, the RegRNA currently has 21,643 known miRNA sequences. During the miRNA prediction, the lower MFE values reveal the energetically more probable hybridizations between the miRNAs and target genes.

TargetScanHuman version 6.2 (Lewis et al., 2005; Grimson et al., 2007) is a web server for predicting miRNAs by searching for the presence of conserved sites that match the seed region of each miRNA. In mammals, predictions are ranked based on the predicted efficacy of targeting as calculated using the context+scores of the sites and their probability of conserved targeting. TargetScanHuman considers matches to annotate human UTRs and their orthologs, as defined by UCSC whole-genome alignments. To predict miRNAs, the name of the target mRNA and species were used as the input. The lower context+score and higher probability of conserved target reveal more probable candidate miRNAs.

\section{MiRNA selection criteria}

The predicted miRNAs from the previous step followed by the selection criteria can provide candidate miRNAs in the next step. The candidate miRNA selection criteria are as follows: 1) exhibited greater than or equal to 2 in 3 prediction tools, 2) high negative free energy that represented more probable hybridizations of miRNA-mRNA duplex, 3) high negative mirSVR score showed a high probability of target inhibition, and 4) high negative context+score and high probability of conserved target revealed good candidate miRNAs for target gene inhibition.

\section{Target accessibility}

The miRNAs that were qualified by the candidate miRNA selection criteria were assessed in terms of target accessibility using Sfold. The Sfold software is a statistical sampling algorithm for predicting the RNA secondary structure that is accessible for RNA-targeting nucleic acids through base-pairing interactions. Target accessibility was predicted by probability; sites with high probability are estimated to be a good accessible region for miRNA binding (Ding and Lawrence, 2001, 2003; Ding et al., 2004). The miRNAs that can hybridize to target regions of mRNA were available as candidates for target gene inhibition. 


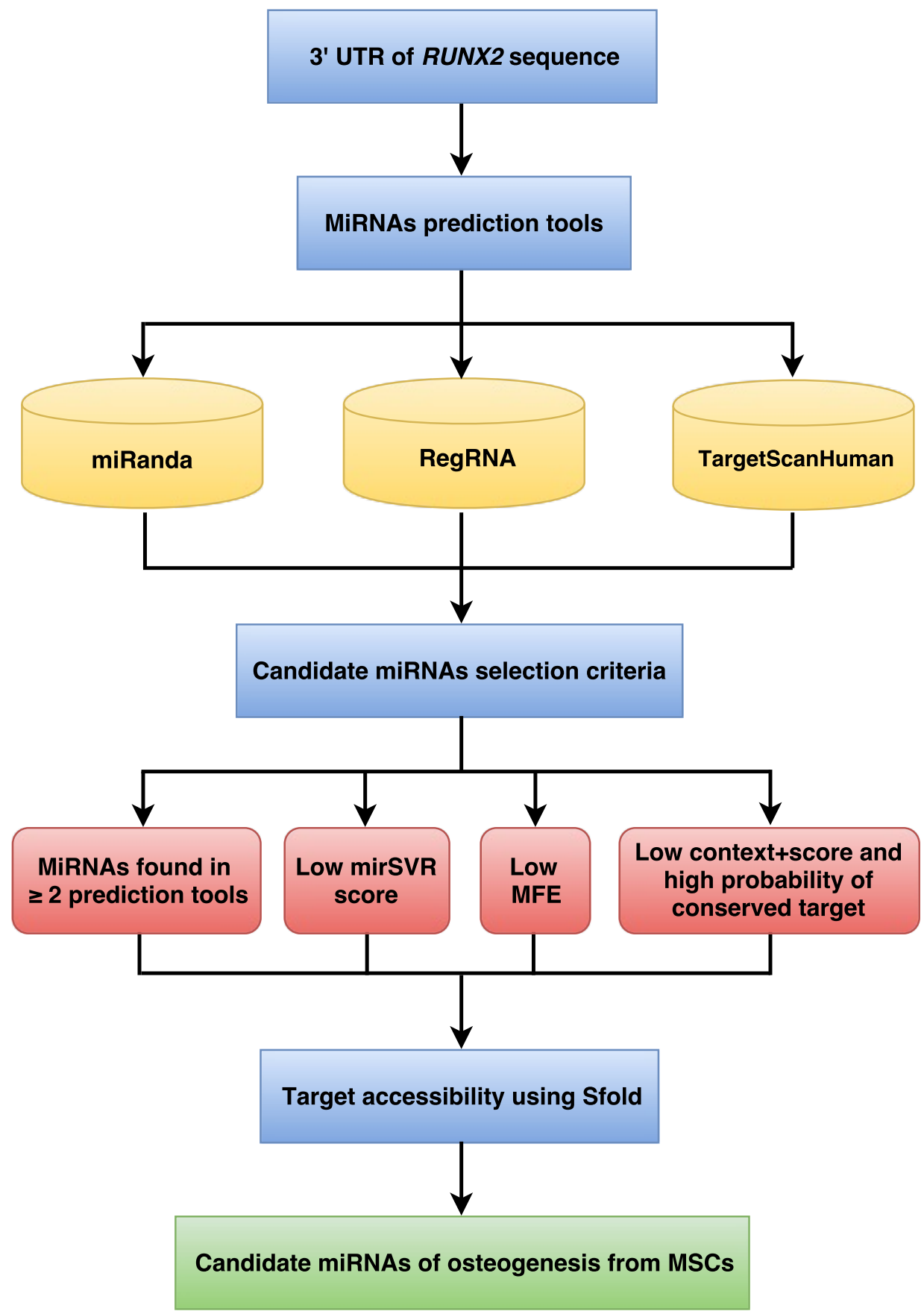

Figure 1. Schematic representation of the workflow for the identification of miRNAs involved in osteogenesis.

\section{RESULTS}

\section{Prediction of RUNX2-specific miRNAs}

Following the implementation of the workflow for miRNA prediction that was explained in the materials and methods (Figure 1), to identify the predicted miRNAs of the RUNX2 gene, we computationally identified miRNAs using three prediction tools, namely, miRanda, RegRNA and TargetScanHuman. The 3'UTR sequence of RUNX2 was retrieved from the NCBI database, which is 3,777 nucleotides in length. Then, miRNAs were predicted using miRNA prediction tools. The results indicated that 922,71 and 30 miRNAs were predicted by RegRNA, miRanda and TargetScanHuman, respectively (Supplemental Table 
S2).

\section{Selection of $R U N X 2$ miRNAs}

The predicted miRNAs will be manually selected for further candidate miRNAs. Based on the selection criteria that were explained in the materials and methods, we retrieved only 27 miRNAs from all three prediction tools that satisfied all criteria. The selected miRNAs that resulted from at least two prediction tools and that matched all remaining criteria were used. The results showed 40 miRNAs from overlapping between RegRNA and miRanda, 2 miRNAs from overlapping between RegRNA and TargetScan, and 1 miRNA from overlapping between miRanda and TargetScan. The list of these selected miRNAs and their respective MFE values are presented in Figure 2. Then, we categorized 70 miRNAs in two tables: those that resulted from all three prediction tools (Supplemental Table S3) and those that resulted from 2 of 3 prediction tools (Supplemental Table S4), which are sorted by their high negative free energies. Minimal free energy was further used for the determination of candidate miRNAs based on the minimal free energy: $\leq-10$ and $\leq-15 \mathrm{kcal} / \mathrm{mol}$ for miRNAs that were predicted using all three prediction tools and those that were predicted using two prediction tools, respectively. The list of 29 selected miRNAs and their respective values (Figure 2) were further analyzed using the Sfold software.

\section{RUNX2-specific miRNA candidates}

Candidate miRNAs from the prediction were evaluated based on the probability of target accessibility using the Sfold software. The analysis of the predicted target structural accessibility also revealed cases of specific miRNA binding that is important for target gene inhibition. A high probability will increase the chance of successful miRNA binding. We input the 3' UTR of the RUNX2 sequence, and then we obtained a histogram of the probability profile in each nucleotide position (Figure 3 ). The positions of nucleotides with a probability greater than 0.5 were designated to be an accessible region for miRNA binding. Consequently, miRNAs 23b, 23a, 30b, 143, 203, 217, and 221 were selected as potential RUNX2-specific miRNA candidates for target gene inhibition (Figure 4). 


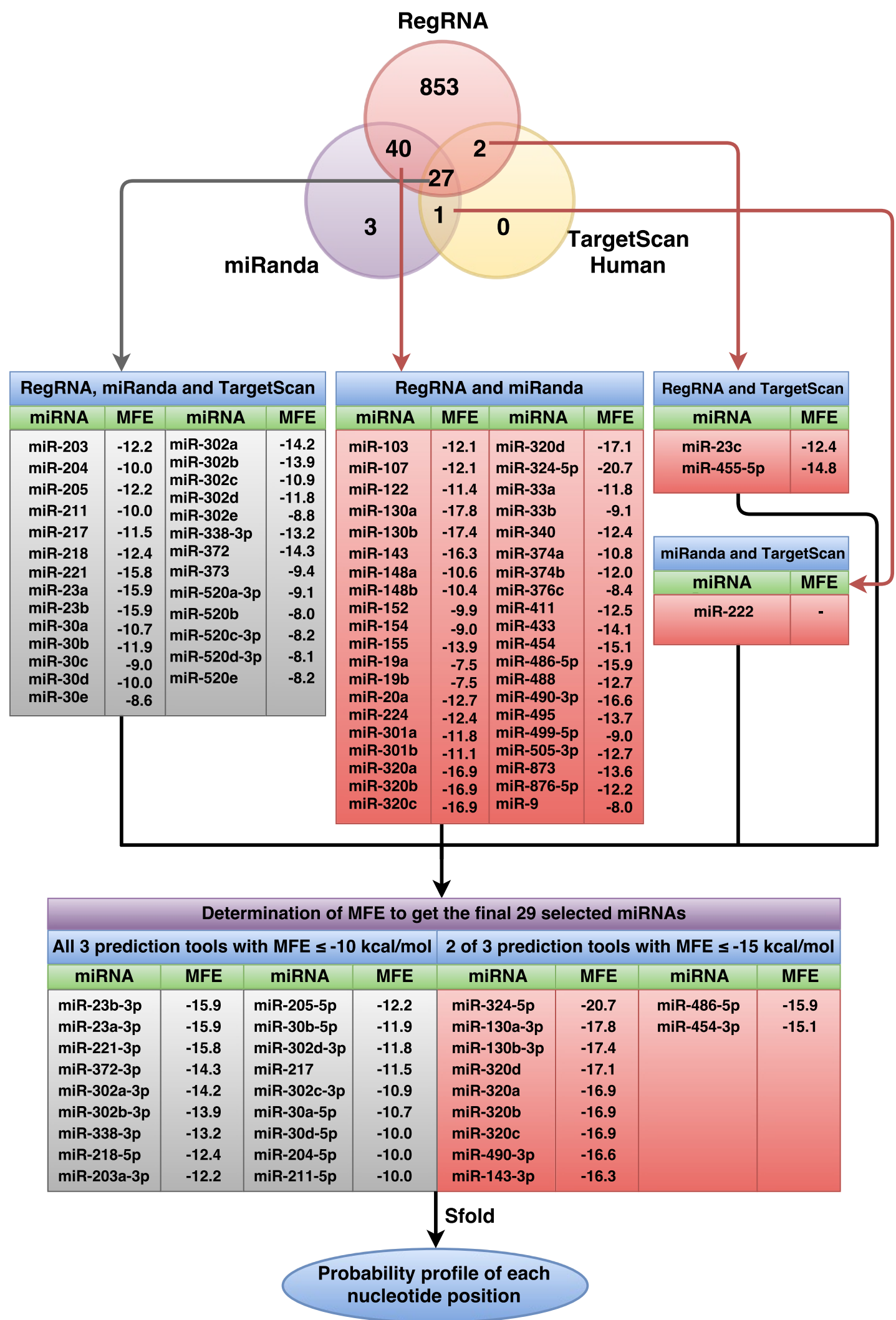

Figure 2. Overall number of $R U N X 2$-specific miRNAs using RegRNA, miRanda, and TargetScanHuman and the respective number of miRNAs obtained from the prediction tools. All RUNX2-specific miRNAs with their respective MFE values are shown in the upper table. MiRNAs were further filtered as to derive the final set of 29 selected miRNAs (shown in the lower table) using MFE thresholds of $\leq-10$ (all 3 prediction tools) and $\leq-15$ ( 2 of 3 prediction tools) $\mathrm{kcal} / \mathrm{mol}$. 
Probability Profile of Target RNA (from position 1 to 3753)

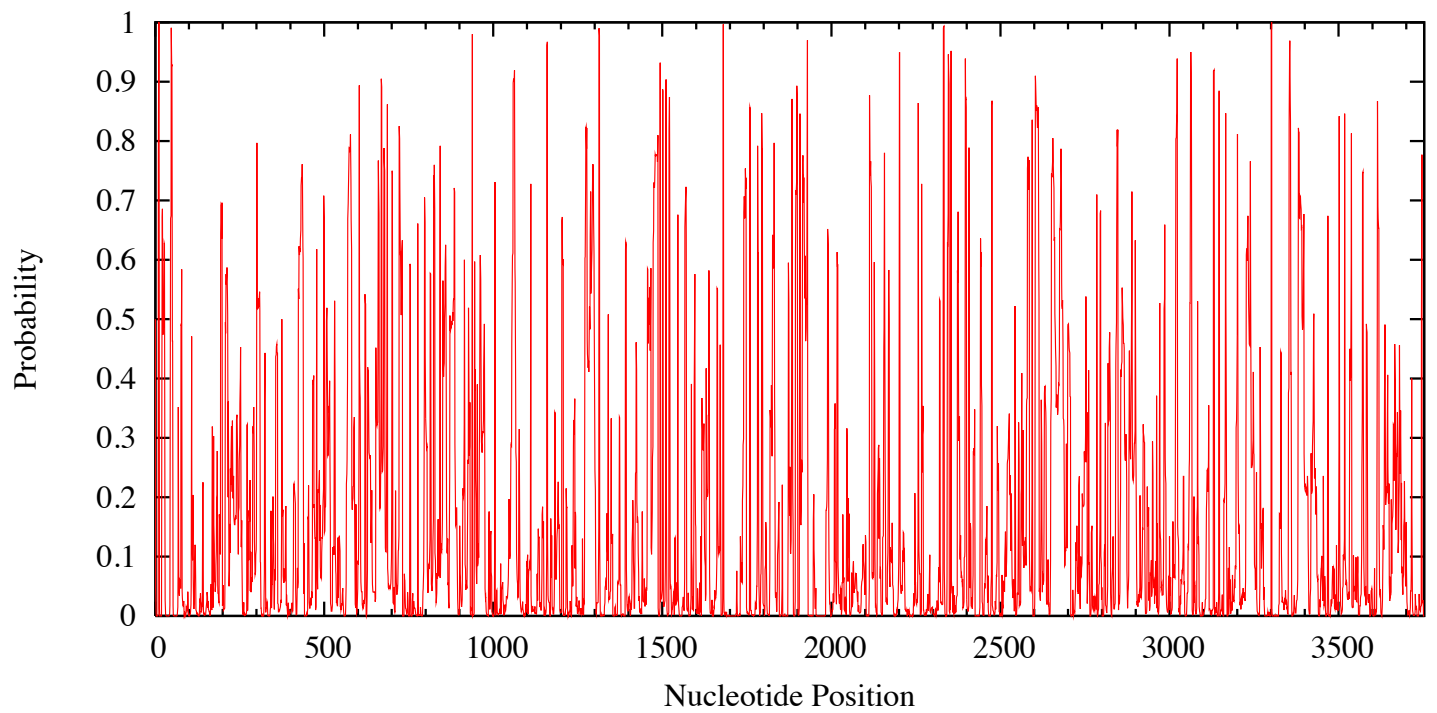

Figure 3. Probability profile of 3'UTR RUNX2 target RNA. The target regions are indicated on the histogram. Target structure features are essential for target binding by miRNA. The higher the probability, the stronger the miRNA binding to the secondary structure of the target region. 

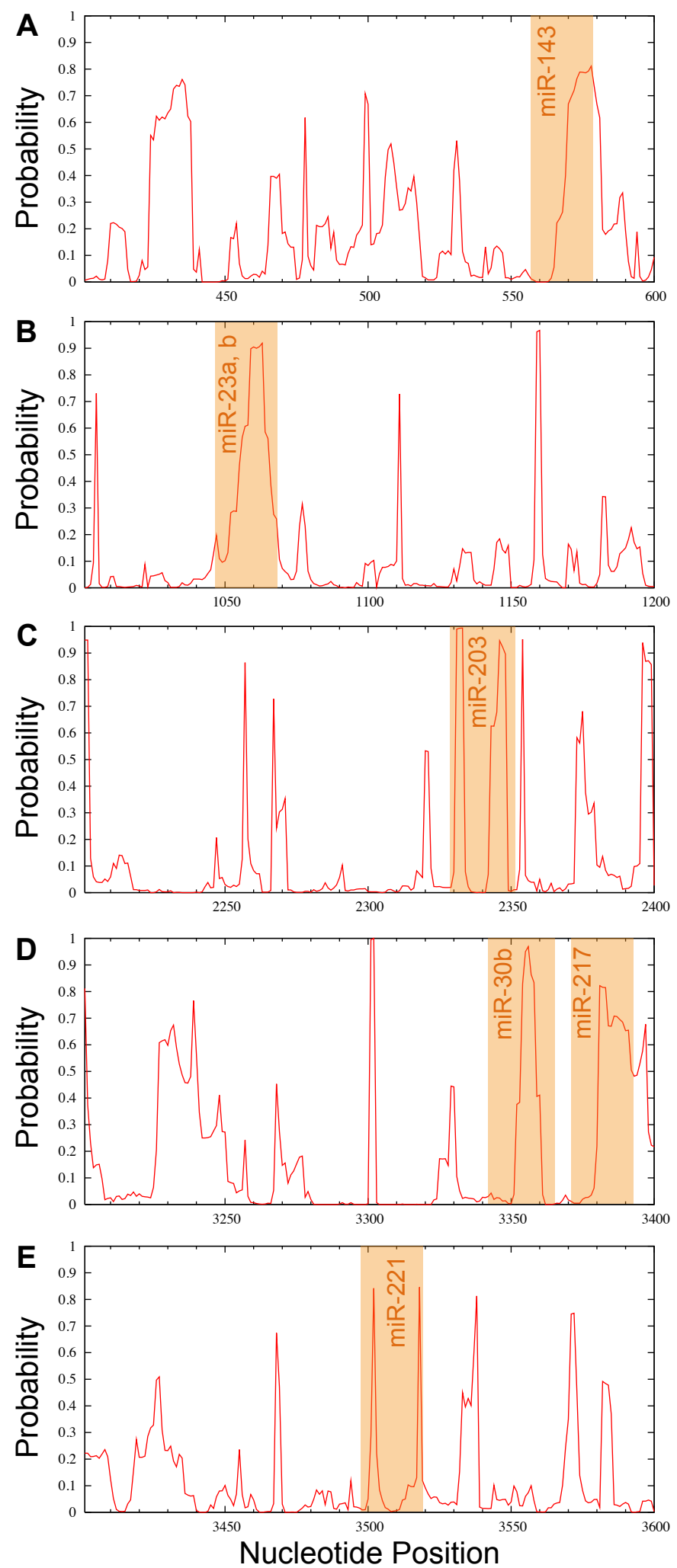

Figure 4. Probability profile of the miRNA binding region of the RUNX2 target RNA. Binding regions are shown by the orange shaded regions for miR-143 (A), miR-23a, b (B), miR-203 (C), miR-30b and miR-217 (D) as well as miR-221 (E) at positions 558 to 578, 1044 to 1068, 2329 to 2352, 3343 to 3366 and 3371 to 3393, as well as 3497 to 3519 , respectively. A probability over 0.5 in the complementary seed sequence was denoted as the accessible region for miRNA binding. 


\section{DISCUSSION}

RUNX2 is a transcription factor or master switch that controls osteogenesis, including MSC condensation, osteoblast proliferation and differentiation, and osteoblast maturation (Komori, 2002). There are multiple mechanisms that control RUNX2 activity, including transcriptional regulation, translational modifications, alternative splicing, subnuclear localization, and interactions with cofactors, among others (Stein et al., 2004). RUNX2 is specifically expressed in the early stage of osteoblast differentiation (Pratap et al., 2003). Overexpression of $R U N X 2$ or $C b f a-1$ (core binding factor $\alpha 1$ ) in MC3T3-E1 preosteoblastic cells showed increased expression of bone marker genes, including collagen type 1, osteopontin, and bone sialoprotein (Ducy et al., 1997). A recent study showed that $R U N X 2$ or $C b f a-1$ null mice can induced a complete lack of bone formation because of osteoblast maturational arrest, indicating a role of RUNX2 as an osteoblast-specific transcription factor (Komori et al., 1997). The process of osteogenesis occurring depends on the expression of key transcriptional factors and changes in the epigenetic mechanisms. Epigenetic regulation of osteogenesis consists of DNA methylation, histone modification, and miRNA regulation, and they can influence gene expression by turning on or off the specific regulatory genes. Villagra et al. (2002) have provided evidence that the expression of the bone-specific rat osteocalcin gene can be regulated via DNA methylation.

MiRNAs have an important role in several cellular processes, such as development, cell proliferation, and cell death (Friedman and Jones, 2009). The expression of miRNAs is associated with several health problems, including osteoporosis (van Wijnen et al., 2013). Therefore, studying a functional miRNA to regulate gene expression in bone development is still challenging for use in diagnostic and therapeutic approaches. MiRNAs showed a regulatory role in the osteogenesis of MSCs by regulating various biological processes, including inhibition of protein translation and promotion of mRNA degradation. Several studies have used microarrays to characterize the genes and expression profiles of miRNAs involved in osteogenesis. These studies identified the miRNA expression profile and their targeted gene in differentiated and undifferentiated hMSCs. They reported that miR-335 is downregulated in hMSC differentiation of adipogenesis and osteogenesis (Tome et al., 2011). Huang et al. (2012) applied microarrays to analyze miRNA expression profiles during adipogenic and osteogenic differentiation from hADMSCs. The results from the microarray analysis revealed that miR-22 decreased in adipogenesis but increased in osteogenesis, thus indicating a positive role of miR-22 in the regulation of osteogenesis. Microarrays can be used to identify the expression levels of thousands genes with high sensitivity and specificity, but it has limitations with respect to expense and difficulty in data interpretation. Thus, bioinformatics tools are powerful tools for investigating of genes and miRNA profiles. Effectively predicting miRNA-mRNA interactions remains challenging due to the complex process and limited knowledge of the interactions. Therefore, using bioinformatics tools is necessary for predicting miRNAs to find possible miRNA-mRNA interactions.

A variety of miRNA prediction algorithms are available, as well as different approaches. In this study, we used online bioinformatics tools, namely, RegRNA, miRanda, and TargetScan, to predict miRNAs that mediate posttranscriptional control of RUNX2 expression involved in the process of osteogenesis. RegRNA is widely used for the prediction of functional RNA motifs because the RegRNA database is always updated, particularly the identification tool. This software also has a user-friendly interface, is easy to use, and provides good graphical visualization. miRanda is an algorithm for finding genomic targets of miRNAs by presenting mirSVR scores, which are calculated from 3 factors: binding energy of miRNA-miRNA interactions, conservation of the entire target site and the 3'UTR region. This algorithm is beneficial for predicting imperfect binding within the seed region, but it has low precision and many false positives as estimated by experimental results (Alexiou et al., 2009; John et al., 2004). TargetScan is frequently used for miRNA prediction because of its convenience. This algorithm predicts miRNAs complementary with conserved sites of 3'UTR regions. The search result is limited only to the sites that are perfectly complementary in the miRNA seed sequence and extended to 22 nucleotide-long sequences that represent true interactions. In addition, there are many parameters for determining the outcome score, including seed match, complementary outside the seed sequence and positioning contribution; however, sites with poor seed pairing are missed (Lewis et al., 2005). We selected three prediction tools because the combination of three prediction tools would be helpful in decreasing false positives and false negatives and provide high accuracy and precision for the selection of specific miRNAs.

All miRNAs predicted to target the 3'UTR of RUNX2 mRNA are 23b, 23a, 30b, 143, 203, 217, and 221, and these might be potential miRNAs in osteogenesis from MSCs because these miRNAs have high 
negative free energies, thus representing a high probability of miRNA and mRNA interactions. Moreover, the positions of the target region that miRNAs can hybridize have a probability greater than 0.5 , indicating that these miRNAs potentially play a regulatory role in osteogenesis. Interestingly, our candidate miRNAs have previously been shown to have some association with osteogenesis. The expression of the miR-30 family was examined during osteogenesis using miRNA PCR arrays. They found that the miR-30 family could repress $R U N X 2$ mRNA by immediate reduction and rapid recovery during osteogenic differentiation (Eguchi et al., 2013). MiR-221 was investigated using a miRNA microarray, which regulated osteogenic lineage commitment. Down-regulation of miR-221 using anti-miR-221 in hMSCs showed increased osteogenic marker genes but no significant change in the translation of osteopontin and osteocalcin. The results of this study revealed that miR-221 could play an important role in osteogenesis (Bakhshandeh et al., 2012b,a). In addition, miR-204, miR-211 and miR-338-3p, which were predicted in the 3 software packages, were correlated in a previous study. They found that miR-204 and miR-211 were negative regulators of RUNX2, which inhibit osteogenesis and promote adipogenesis of mesenchymal stem cells (Huang et al., 2010). Furthermore, the role of miR-338-3p in human ovarian epithelial carcinoma (EOC) was reported to inhibit ovarian cancer cell growth by targeting RUNX2 (Wen et al., 2015). However, miR-204, miR-221 and miR-338-3p are not candidate miRNAs in this study because they were excluded because of their low probability of target accessibility. A plausible explanation could be that no single miRNA are deemed to be the sole driver for activating a gene but that the orchestrated work amongst several miRNAs is crucial for modulating the target gene. Particularly, Wu et al. (2010) found that 28 of 266 miRNAs inhibited the expression of $\mathrm{p21Cip1/Wafl,} \mathrm{which} \mathrm{is} \mathrm{a} \mathrm{master} \mathrm{downstream} \mathrm{effector} \mathrm{of} \mathrm{tumor}$ suppressors. In addition, Jiang et al. (2009) employed a resemble method in which luciferase reporter assay was used to examine a number of miRNAs that inhibit the proto-oncogene, cyclin Dl. They found that 7 significant miRNAs could suppress the 3'UTR activity of cyclin D1. Moreover, several factors such as Watson-Crick base pairing, energy and seed region of miRNA, are essential for controlling the in vivo interaction of miRNA and mRNA. In summary, in order to achieve a high confidence and accuracy set of candidate miRNAs which can affect the target gene, the strict selection criteria employed herein should be applied, especially for further experimental validation. However, the criterion could be made more or less stringent by the researcher depending on the circumstances or purpose of an investigation of interest, which would directly determine the breadth of miRNAs identified from the investigation.

Thus, the workflow for identifying miRNAs using bioinformatics tools is an alternative approach that is highly useful for the prediction of novel miRNAs in osteogenesis rather than using microarrays. This technique is convenient and inexpensive. For future studies, gene-targeting miRNAs and miRNA expression in vitro and in vivo are highly needed to evaluate the accuracy and precision of the identification of miRNAs.

\section{CONCLUSION}

Osteogenesis is a very important process in the human body, which encompasses several cellular processes. The differentiation of MSCs to preosteoblasts is considered to be a critical step in osteogenesis. This step is controlled by many regulatory mechanisms, i.e., transcription factors, signaling pathways, and epigenetic mechanisms. MiRNAs showed a regulatory role in osteoblastic differentiation by regulating gene expression during the post-transcriptional process. A bioinformatics tool is a good choice for predicting candidate miRNAs involved in osteogenesis. Using three miRNA prediction tools in combination with selection criteria, we selected RUNX2 as a target because the RUNX2 transcription factor acts as a master switch controlling osteogenesis. We retrieved 7 candidate miRNAs, including miR-23b, 23a, 30b, 143, 203, 217, and 221. The knowledge obtained from this study can provide basic information regarding miRNAs in osteogenesis; however, further studies are needed to evaluate miRNAs and target genes in vitro and in vivo for clarifying the complex mechanism of osteogenesis.

\section{ACKNOWLEDGMENTS}

This research project is supported by Mahidol University. 


\section{REFERENCES}

Alexiou, P., Maragkakis, M., Papadopoulos, G., Reczko, M., and Hatzigeorgiou, A. (2009). Lost in translation: an assessment and perspective for computational microRNA target identification. Bioinformatics, 25:3049-3055.

Bakhshandeh, B., Hafizi, M., Ghaemi, N., and Soleimani, M. (2012a). Down-regulation of miRNA-221 triggers osteogenic differentiation in human stem cells. Biotechnology Letters, 34:1579-1587.

Bakhshandeh, B., Soleimani, M., Hafizi, M., Paylakhi, S., and Ghaemi, N. (2012b). MicroRNA signature associated with osteogenic lineage commitment. Molecular Biology Reports, 39:7569-7581.

Betel, D., Koppal, A., Agius, P., Sander, C., and Leslie, C. (2010). Comprehensive modeling of microRNA targets predicts functional non-conserved and non-canonical sites. Genome Biology, 11:R90.

Chang, T., Huang, H., Hsu, J., Weng, S., Horng, J., and Huang, H. (2013). An enhanced computational platform for investigating the roles of regulatory RNA and for identifying functional RNA motifs. BMC Bioinformatics, 14:S4.

Deans, R. J. and Moseley, A. B. (2000). Mesenchymal stem cells: biology and potential clinical uses. Experimental Hematology, 28:875-884.

Ding, Y., Chan, C., and Lawrence, C. (2004). Sfold web server for statistical folding and rational design of nucleic acids. Nucleic Acids Research, 32:W135-141.

Ding, Y. and Lawrence, C. (2001). Statistical prediction of single-stranded regions in RNA secondary structure and application to predicting effective antisense target sites and beyond. Nucleic Acids Research, 29:1034-1046.

Ding, Y. and Lawrence, C. (2003). A statistical sampling algorithm for RNA secondary structure prediction. Nucleic Acids Research, 31:7280-7301.

Ducy, P., Zhang, R., Geoffroy, V., Ridall, A., and Karsenty, G. (1997). Osf2/Cbfa1: a transcriptional activator of osteoblast differentiation. Cell, 89:747-754.

Eguchi, T., Watanabe, K., Hara, E., Ono, M., Kuboki, T., and Calderwood, S. (2013). OstemiR: a novel panel of microRNA biomarkers in osteoblastic and osteocytic differentiation from mesencymal stem cells. PLoS One, 8:e58796.

Erices, A., Conget, P., and Minguell, J. J. (2000). Mesenchymal progenitor cells in human umbilical cord blood. British Journal of Haematology, 109:235-242.

Friedman, J. and Jones, P. (2009). MicroRNAs: critical mediators of differentiation, development and disease. Swiss Medical Weekly, 139:466-472.

Grimson, A., Farh, K., Johnston, W., Garrett-Engele, P., Lim, L., and Bartel, D. (2007). MicroRNA targeting specificity in mammals: determinants beyond seed pairing. Molecular Cell, 27:91-105.

Hayrapetyan, A., Jansen, J. A., and van den Beucken, J. J. (2015). Signaling pathways involved in osteogenesis and their application for bone regenerative medicine. Tissue Engineering Part B Reviews, 21:75-87.

He, L. and Hannon, G. J. (2004). MicroRNAs: small RNAs with a big role in gene regulation. Nature Reviews Genetics, 5:522-531.

Huang, H., Chien, C., Jen, K., and Huang, H. (2006). RegRNA: an integrated web server for identifying regulatory RNA motifs and elements. Nucleic Acids Research, 34:W429-34.

Huang, J., Zhao, L., Xing, L., and Chen, D. (2010). MicroRNA-204 regulates Runx2 protein expression and mesenchymal progenitor cell differentiation. Stem Cells, 28:357-364.

Huang, S., Wang, S., Bian, C., Yang, Z., Zhou, H., and Zeng, Y. (2012). Upregulation of miR-22 promotes osteogenic differentiation and inhibits adipogenic differentiation of human adipose tissue-derived mesenchymal stem cells by repressing HDAC6 protein expression. Stem Cells and Development, 21:2531-2540.

James, A. W. (2013). Review of Signaling Pathways Governing MSC Osteogenic and Adipogenic Differentiation. Scientifica (Cairo), 2013:684736.

Jiang, Q., Feng, M. G., and Mo, Y. Y. (2009). Systematic validation of predicted microRNAs for cyclin D1. BMC Cancer, 9:194.

John, B., Enright, A., Aravin, A., Tuschl, T., Sander, C., and Marks, D. (2004). Human MicroRNA targets. PLoS Biology, 2:e363.

Kang, H. and Hata, A. (2015). The role of microRNAs in cell fate determination of mesenchymal stem cells: balancing adipogenesis and osteogenesis. BMB reports, 48:319-323.

Komori, T. (2002). Runx2, a multifunctional transcription factor in skeletal development. Journal of 
Cellular Biochemistry, 87:1-8.

Komori, T. (2006). Regulation of osteoblast differentiation by transcription factors. Journal of Cellular Biochemistry, 99:1233-1239.

Komori, T., Yagi, H., Nomura, S., Yamaguchi, A., Sasaki, K., and Deguchi, K. (1997). Targeted disruption of Cbfa1 results in a complete lack of bone formation owing to maturational arrest of osteoblasts. Cell, 89:755-764.

Lewis, B., Burge, C., and Bartel, D. (2005). Conserved seed pairing, often flanked by adenosines, indicates that thousands of human genes are microRNA targets. Cell, 120:15-20.

Pittenger, M. F., Mackay, A. M., Beck, S. C., Jaiswal, R. K., Douglas, R., Mosca, J. D., Moorman, M. A., Simonetti, D. W., Craig, S., and Marshak, D. R. (1999). Multilineage potential of adult human mesenchymal stem cells. Science, 284:143-147.

Pratap, J., Galindo, M., Zaidi, S., Vradii, D., Bhat, B., and Robinson, J. (2003). Cell growth regulatory role of Runx2 during proliferative expansion of preosteoblasts. Cancer Research, 63:5357-5362.

Stein, G. S., Lian, J. B., van Wijnen, A. J., Stein, J. L., Montecino, M., Javed, A., Zaidi, S. K., Young, D. W., Choi, J. Y., and Pockwinse, S. M. (2004). Runx2 control of organization, assembly and activity of the regulatory machinery for skeletal gene expression. Oncogene, 23:4315-4329.

Tome, M., Lopez-Romero, P., Albo, C., Sepulveda, J., Fernandez-Gutierrez, B., and Dopazo, A. (2011). miR-335 orchestrates cell proliferation, migration and differentiation in human mesenchymal stem cells. Cell Death and Differentiation, 18:985-995.

van Wijnen, A., van de Peppel, J., van Leeuwen, J., Lian, J., Stein, G., and Westendorf, J. (2013). MicroRNA functions in osteogenesis and dysfunctions in osteoporosis. Current Osteoporosis Reports, 11:72-82.

Villagra, A., Gutierrez, J., Paredes, R., Sierra, J., Puchi, M., and Imschenetzky, M. (2002). Reduced CpG methylation is associated with transcriptional activation of the bone-specific rat osteocalcin gene in osteoblasts. Journal of Cellular Biochemistry, 85:112-122.

Wen, C., Liu, X., Ma, H., Zhang, W., and Li, H. (2015). miR3383p suppresses tumor growth of ovarian epithelial carcinoma by targeting Runx2. International Journal of Oncology, 46:2277-2285.

Wu, S., Huang, S., Ding, J., Zhao, Y., Liang, L., Liu, T., Zhan, R., and He, X. (2010). Multiple microRNAs modulate p21Cip1/Waf1 expression by directly targeting its 3' untranslated region. Oncogene, 29:2302 2308.

Zhang, J., Fu, W., He, M., Wang, H., Wang, W., and Yu, S. (2011). MiR-637 maintains the balance between adipocytes and osteoblasts by directly targeting Osterix. Molecular Biology of the Cell, 22:3955-3961.

Zuo, B., Zhu, J., Li, J., Wang, C., Zhao, X., and Cai, G. (2015). microRNA-103a functions as a mechnosensitive microRNA to inhibit bone formation through targeting Runx2. Journal of Bone and Mineral Research, 30:330-345. 